Șerban Al., Obadă B., Turcu R., Anderlik St., Botnaru V.

\title{
Distal tibial fracture treated by minimally invasive plate osteosynthesis after external fixation Retrosnective clinical and radiographic assessment
}

Clinic of Orthopedics and Traumatology, Emergency County Hospital Constanța

\begin{abstract}
Fractures of the horizontal surface of the distal tibia are known commonly as pylon or plafond fractures, and represent $1-5 \%$ of lower extremity fractures, $7-10 \%$ of all tibial fractures. The protocol consisted of immediate (within eight to 24 hours) open reduction and internal fixation of the fibula, using a fibular plate or one third tubular plate and application of an external fixator spanning the ankle joint. In the second stage, the treatment of proximal and distal tibial fractures with close reduction and MIPPO technique can preserve soft tissue, simplify operative procedure and decrease wound, obtain rigid internal fixation and guarantee early function exercises of ankle joint. In this study we evaluated 22 patients treated in Clinical Emergency Hospital Constanta between April 2012 - July 2013 diagnosed with multifragmentary fractures of the distal tibia. This study evaluates the treatment of complex fractures of distal tibia with locked plate after external fixation. There were 17 males and 5 females of mean age 51,7 years (31-68). The mean follow-up period was 14 weeks. (Ranging from 9-16 weeks). All patients were fully weight bearing at 16 weeks (ranging 9-16 weeks) showing radiological union. There were no cases
\end{abstract} Dr. Obadă Bogdan

bogdanobada@yahoo.com of failures of fixation, or rotational misalignment. No significant complication was observed in our patients. MIPO is an effective method of treatment for distal tibial fractures, reduce surgical trauma and maintain a more biologically favorable environment for fracture healing, reducing risks of infection and nonunion.

Keywords: external fixation, locking compression plate (LCP); minimally invasive percutaneous plate osteosynthesis (MIPPO), image intensifier,

\section{Introduction}

Fractures of the horizontal surface of the distal tibia are known commonly as pilon or plafond fractures. They represent $1-5 \%$ of lower extremity fractures and $7-10 \%$ of all tibial fractures [1].

The injury is an intra-articular fracture of the tibiotalar joint with varying degrees of proximal extension into the tibial metaphysis. They are the result of an axial load on the tibia, with or without an accompanying rotational force. The aim of operative treatment is to anatomically reduce the fracture fragments to restore the congruity of the joint surface and promote bony union with minimal disruption of 
the soft tissue envelope [2].

The soft tissues around the ankle and distal tibia are easily compromised by trauma and subsequent operative fracture treatment [1,2]. Salvage of these challenging problems more often than not requires a staged treatment based on thorough debridement(s), antibiotic treatment until infection is eliminated followed by reconstruction [3-8]. The initial debridement should include removal of all failed hardware. The resulting instability will compromise the eradication of infection. To prevent this, temporary bony stabilization can be obtained by some form of external fixation. However, most external frames for the lower leg are bulky and cumbersome for the patient, leading to problems with sleeping, clothing, and can cause an impediment to the contralateral extremity when walking [3].

Optimum repair of the articular damage demands precise anatomical reduction accompanied by early motion. Achieving this ideal, however, is often hindered by factors such as loss of bone mass, infection and partial thickness skin or wound necrosis, which makes surgical treatment difficult or results in failure and can contribute to a less than optimal reconstruction that may predispose the ankle joint to the development of post-traumatic arthrosis, nonunion, malunion or pseudarthrosis.

The issue of localized devitalization of bone and further damage to surrounding soft tissue may occur after internal fixation. In addition, external fixation was introduced to treat pylon fractures as an alternative because it could reduce most of the abovementioned complications at a certain level.

The two-staged procedure protocol-use of the external fixator in the first stage and the internal fixator in the second stage-has been applied in the treatment of pilon fractures for many years in different countries and has been widely accepted by many orthopaedic surgeons [4].

High-energy pylon fractures should be temporized with an external fixator with fibular plating to restore length. Definitive fixation should be planned for between 10 and 14 days, by which time the soft tissue envelop is likely to be ready to accept the further insult of surgery [1].

Minimal disturbance of the soft tissue envelope is key to the prevention of the common wound problems of dehiscence and infection. The vascularity of the soft tissue sleeve surrounding the distal tibia is tenuous and aggressive handling with extensive periosteal stripping will disturb the nutrition to the myocutaneous tissue and underlying bone [2].

Treatment of proximal and distal tibial fractures with close reduction and MIPPO technique can preserve soft tissue, simplify operative procedure and decrease wound, obtain rigid internal fixation and guarantee early function exercises of ankle joint. The method has the advantages of less soft tissue injury, less blood loss, reliable fixation, which is effective method in treating distal tibial fractures and corresponds with the standpoint of biological fixation [5].

\section{Material and Methods}

From April of 2012 to July 2013, 22 patients (17 males and 5 females - Figure 1) with complex fracture of distal tibia and fibula were treated with locked compression plate by minimally invasive method, after external fixation and plating of fibula fractures.

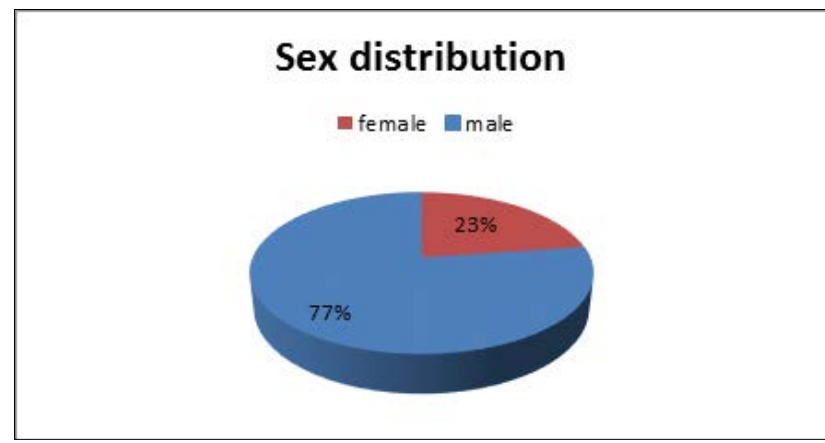

Figure 1

All patients developed fractures due to high energy trauma (a motor vehicle accident, falling from the height, sport accident, work accident) (Figure 2) and all underwent surgery for treatment of fracture soon after a systemic condition that was stabilized. 
Our patients were 31 to 68 years old (mean: 51.7 ) (Figure 3) 9 patients had right sided fracture and 13 had a left sided one.

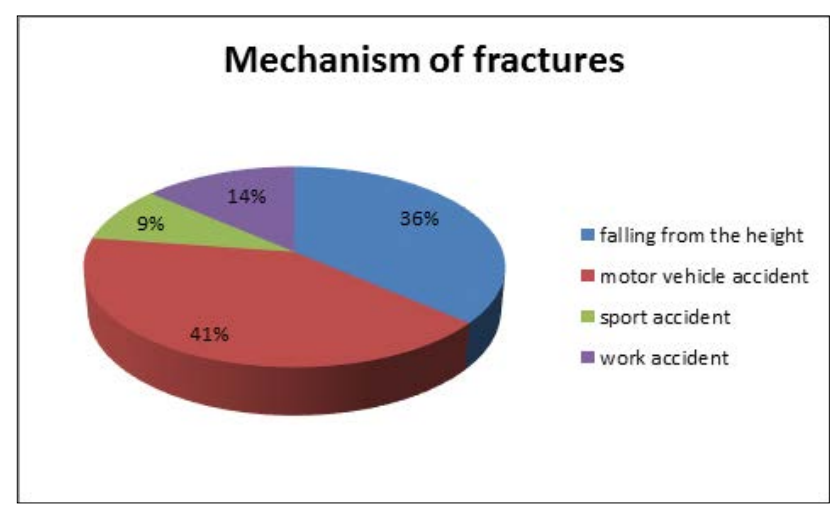

Figure 2

All patients underwent osteosynthesis of fibula (1/3 semitubular plate) through the lateral approach, and stabilization of ankle with external fixator, as soon as general condition of patients was stabilized. Initial fibula fixation provides lengthening and helps place the tibia in the correct position.

In the second stage, the articular fragments were anatomically reduced by percutaneous method, utilizing fluoroscopy and pointed reduction forceps. Once articular reduction was achieved, if possible, the articular fragments were stabilized with lag screws. The appropriate length of the plate was determined by preoperative planning.

Cases were operated on standard operating tables, in the supine position with air tourniquet application following elevation of the extremity.

A 2-3 cm incision was made along the anteromedial aspect of the tibia, proximal to the fracture and distally at the level of the medial malleolus. Typically, a subcutaneous tunnel was created between the two incisions and along the medial aspect of the tibia by blunt dissection using a periosteal elevator. The screws were then placed at each end of the plate through the two incisions and in the mid position via small percutaneous stab incisions.

Post operative check X-ray was taken to assess the reduction. Parenteral antibiotics (Inj. ceftriaxone + Inj. amikacin) were given for two days followed by oral antibiotics depending upon the condition of the wound.
On 3rd post-operative day wound was examined and antiseptic dressing done. The splint was removed and physical therapy with quadriceps exercises and gentle active assisted exercises started depending on toleration of patient. Sutures were removed between 10th to 14 th days.

\section{Results}

Road traffic accidents were the commonest mode of trauma of distal tibial fractures. Overall out of 22 cases, in 9 cases the injury was due to road traffic accidents.

In case of distal tibial fractures the mean time for radiological union was 17 weeks with the range being from 14 to 24 weeks.

According to the AO classification; 7 patients $(32 \%)$ were $43 \mathrm{~A} 3,5$ patients $(23 \%) 43 \mathrm{~B} 2,4$ patients (18\%) 43C1, 4 patients (18\%) 43C2, 2 patients $(9 \%)$ 43C3 (Figure 3).

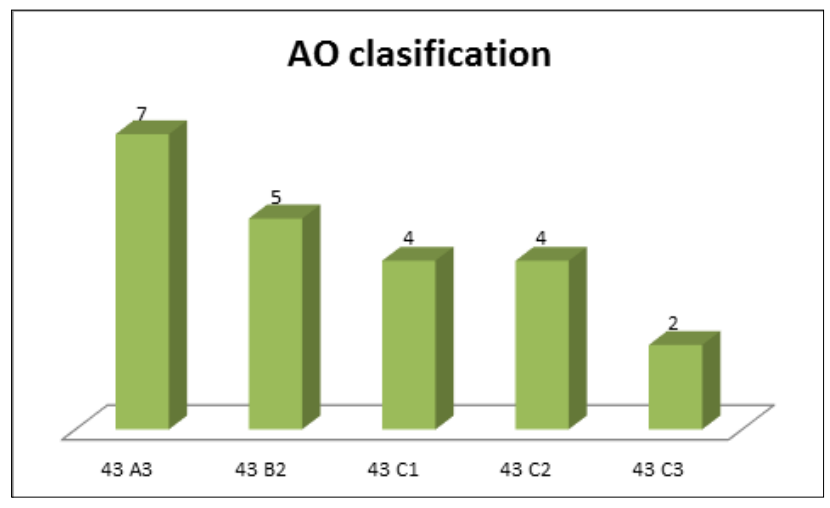

Figure 3

Fractures were caused road traffic accidents 9 cases $(41 \%)$, falling from a height in 8 cases $(36 \%)$, occupational accident in 3 cases (14\%), and sport accidents in 2 cases (9\%) (Figure 2).

Full weight-bearing was allowed after a mean of 14.2 (range: 12 to 20 ) weeks. 
Anteroposterior and lateral radiographs of distal tibial metaphyseal fracture. Follow-up radiographs: first stage - external fixator and fibula plating; second stage: the tibial fracture was stabilized with LCP (MIPPO)
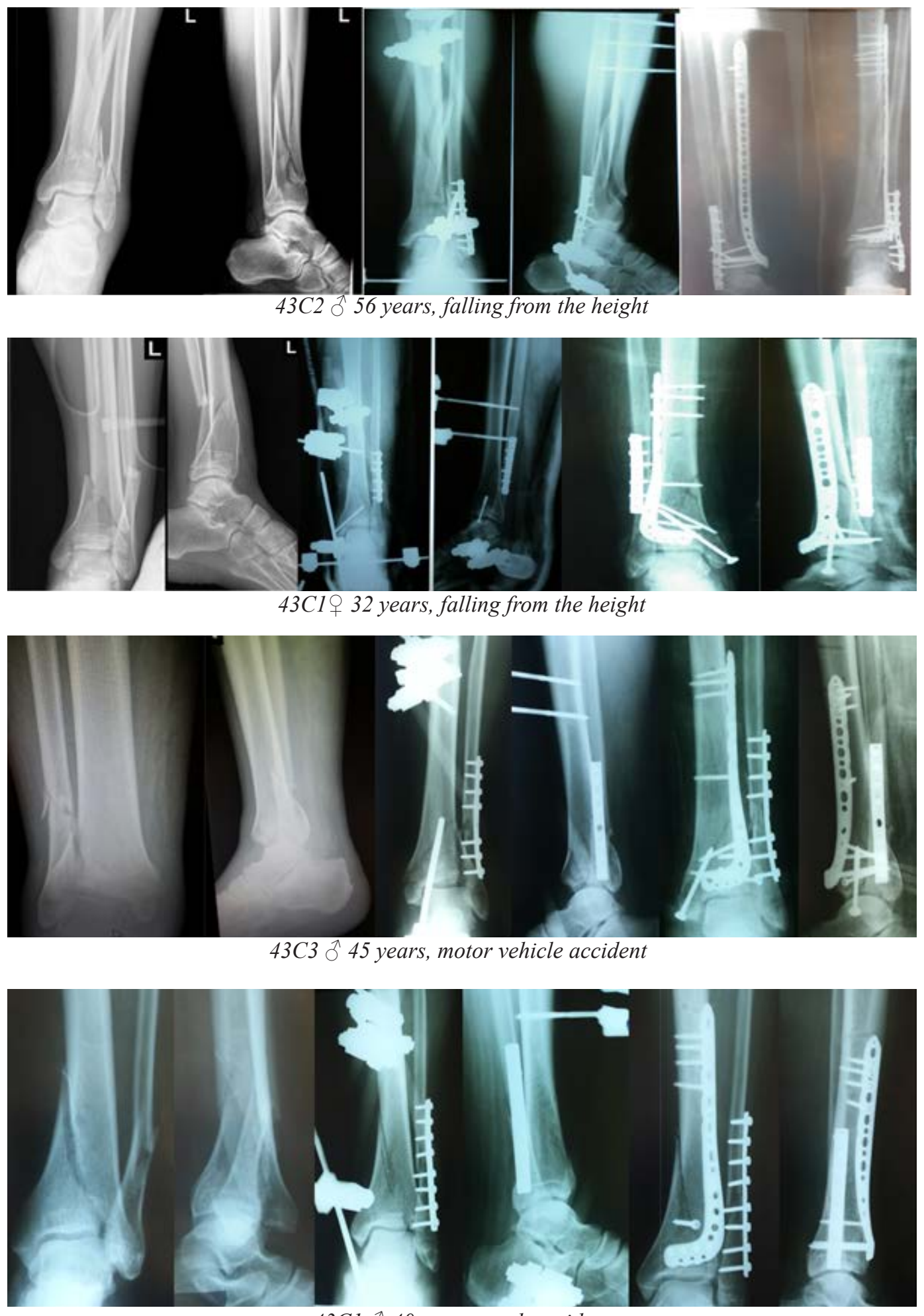

$43 \mathrm{Cl}$ ô 40 years, work accident 


\section{Discussion}

MIPO of these fractures is technically feasible and advantageous in that it minimizes soft tissue compromise and devascularization of the fracture fragments. The procedure includes three important components: closed reduction, minimal soft tissue dissection and stabilization with a long percutaneously inserted plate fixed with a limited number of widespread screws. Even though early intervention is advantageous, it is desirable to delay surgery in the presence of gross local swelling until subsidence of swelling and appearance of the wrinkle sign to ensure good local skin condition prior to surgery [6].

In all cases, radiological evidence of bony union was obtained, and the average bony union time was 17 weeks (range 14 to 24 weeks). There were no signs of angular deformity of the tibia over 50 . There were no signs of shortening of the tibia. The ankle joint function was evaluated during the final followup: eleven cases were excellent, eight cases were good, and the other three cases were fair.

There were no signs of complications, such as nonunion, malunion, deep infection, osteomyelitis, breakage of the clamp screw or plate, or neurovascular injury.

\section{Acknowledgements}

Because open distal tibia fractures are often caused by high-energy damage, extensive soft tissue injury and severe communition of the fracture site are usually observed. Moreover, in many cases, functional disability remains after treatment when the fracture is quite close to the ankle joint; in particular, the medial part of the distal tibia, where is little subcutaneous soft tissue, close to the periosteum. An open wound and extensive soft tissue injury is usually found on the medial side in an open distal tibia fracture; which usually precludes primary skin, and soft tissue, covering. Therefore, in the treatment of fractures, both the stability of the soft tissue covering, and anatomical reduction in the articular surface, need to be obtained. Therefore, there are many difficulties associated with treatment, which are often followed by a staged treatment [7].

Application of the two-stage procedure protocol with MIPPO seems to play a key role in reducing infection rates associated with open reduction and internal fixation of pylon fractures [4].

Initial fibula fixation provides lengthening and helps place the tibia in the correct position.

MIPPO aims to reduce surgical trauma and protect the vascular integrity and osteogenic hematoma of the fracture, and is an effective alternative treatment for distal tibia fractures, with low complication and high union rates [8].

\section{References}

1. Egol K.A., Wolinsky P. \& Koval K.J. (2000). Open reduction and internal fixation of tibial pilon fractures. Foot Ankle Clin 5(4), 873-885

2. McCann P.A., Jackson M., Mitchell S.T. \& Atkins R.M. (2011). Complications of definitive open reduction and internal fixation of pilon fractures of the distal tibia. Int Orthop 35(3), 413-418

3. Tulner S.A., Strackee S.D. \&Kloen P. (2012). Metaphyseal locking compression plate as an external fixator for the distal tibia. Int Orthop. 36(9), 1923-1927

4. Tong, D., Ji.F., Zhang H., Ding W., Wang Y \& Cheng P. (2012). Two-stage procedure protocol for minimally invasive plate osteosynthesis technique in the treatment of the complex pilon fracture. Int Orthop. 36(4), 833-837

5. Liu, Y. W., Y. Kuang, et al. (2013). Close reduction combined with minimally invasive percutaneous plate osteosynthesis for proximal and distal tibial fractures: a report of 56 patients. Zhongguo Gu Shang. 26(3), 248-251

6. Gupta R.K., Rohilla R.K., Sangwan K., Singh 
V. \& Walia S. (2010). Locking plate fixation in distal metaphyseal tibial fractures: series of 79 patients. Int Orthop. 34(8), 1285-1290

7. Sohn, O. J. \& D. H. Kang (2011). Staged protocol in treatment of open distal tibia fracture: using lateral MIPO. Clin Orthop Surg. 3(1), 69-76

8. Aksekili M.A., Celik I., Arslan A.K., Kalkan T. \& Uğurlu M. (2012). The results of minimally invasive percutaneous plate osteosynthesis (MIPPO) in distal and diaphyseal tibial fractures. Acta Orthop Traumatol Turc. 46(3), 161-167 